\title{
Appraisal of a New Gravitational Constant
}

\author{
Sandro Antonelli* \\ 151A, S.P. 57, Accesso a M., 03017 Morolo Italy \\ *Corresponding author: antonelli41@live.it \\ Received May 05, 2015; Revised May 08, 2015; Accepted May 21, 2015
}

\begin{abstract}
The need of extending the theory of relativity has led M.Tailherer to the hypothesis of a new fundamental equation and constant, embodying in a unique wave equation for the graviton the link between gradient of curvature and deformation of metric. As direct continuation of a preceding work, here a new assessment of the constant $\mathrm{S}$ in the Vortex Theory of gravitation is given in a more direct approach than 1st approximation yielding $S$ $=(2.5 \pm 1.2) \mathrm{E}-19 \mathrm{~m}^{-1}$. Issues are concerned fitting by Maple four binary systems data, also allowing to assign a meaningful inertial mass to the graviton (5.5 \pm 2.6$) \mathrm{E}-61 \mathrm{Kg}$ confirming known heuristic bounding. In Appendix an easy way of getting the vortex's gradient formula is shown along with the whole action of the model and the description of the tide effect on a test mass with respect to a x polarized gravitational wave in the case of an asymmetric source.
\end{abstract}

Keywords: Alternative theories to General Relativity (TGR), Gravitational waves, Vorticity Tensor, Binary Star Systems, massive gravity. PACS. 04.30

Cite This Article: Sandro Antonelli, “Appraisal of a New Gravitational Constant.” International Journal of Physics, vol. 3, no. 4 (2015): 139-149. doi: 10.12691/ijp-3-4-1.

\section{Introduction}

It is well-known as TGR is related to some compelling questions leading towards ambiguities [3]: does not it seem a contradiction indeed that a body subject to mere gravitational fields travel a geodesic of space-time losing in the meanwhile gravitational energy? Furthermore, the expression of gravitational damping in the known formula of third derivative of quadrupole moment (cf.eq.(7)), although proven valid by now also for strong fields by the post-Newtonian formalism with so great an approximation [18], on the other hand breaks down the general covariance in favour of linear one, just because the PN $(\mathrm{v} / \mathrm{c})^{2}$ series expansion cannot avoid the lost of the full symmetry of the exact Einstein's Equations. This is demonstrated by the fact that in TGR any scalar quantity has a physical content only if has an invariant character with respect to general transformations of coordinates, but not so gravitational energy fluxed away, seen that it is always possible to choose a suitable frame for which two gravitating bodies are at rest [2].

Hence I think that from such dilemmas one cannot get out without taking the general relativity as only an aspect of an extended theory as proposed recently by the intriguing tensor Vortex gravity model [6], any advance being subordinate to the determination of the constant $S$, a very cumbersome goal related to the evaluation of the following delayed integro-differential equation, nothing but the solution ${ }^{1}$ at great distance of the double curl in

1 The reader should notice the change of notation with respect to [6] to banish any misleading of the skew symmetric Riemann tensor obtained as $C_{\mu \nu}=R_{\mu v \rho \sigma} \epsilon^{\rho \sigma}$ (respectively $R_{\mu \nu}$ in [6] Eq. 3.3; 4.12) with that of Einstein's equations. Here $C_{\mu \nu}$ is meant derived by contraction of the first order estimate of the Riemann tensor as stressed in [1]. curved Space-Time of the main equation recalled shortly in Appendix B (see also [4,5,6]):

$$
\begin{aligned}
K_{\mu \nu} & =\frac{3}{2 \pi^{2} r^{2}} \int\left(R_{\nu \alpha}^{\beta}{ }_{\mu}-\delta_{\mu}^{\beta} R_{\alpha \nu}\right) K_{\beta}^{\alpha} d^{4} \xi+y_{\mu v} \\
y_{\mu \nu} & =-\frac{3}{4 \pi^{2} r^{2} S} \int C_{\mu \alpha}{ }^{\prime \alpha}{ }^{\alpha} d^{4} \xi
\end{aligned}
$$

with

$$
C_{\mu v}=-k \frac{g_{\alpha v}}{4}\left(T_{\beta \mu}-\frac{1}{2} g_{\beta \mu} T\right) \epsilon^{\alpha \beta}, \quad k=\frac{8 \pi G}{c^{4}}
$$

and $\epsilon^{\alpha \beta}=\left[\begin{array}{cccc}0 & 1 & 0 & 0 \\ -1 & 0 & 1 & 0 \\ 0 & -1 & 0 & 1 \\ 0 & 0 & -1 & 0\end{array}\right] \quad$ where

$T_{\mu v}=\delta^{3}\left(x^{\rho}-x_{1}{ }^{\rho}\right) \mathrm{m} 1 \cdot u^{\mu} u^{v}+\delta^{3}\left(x^{\rho}-x^{\rho}\right) \mathrm{m} 2 \cdot u^{\mu} u^{v}=\int d \tau\left[\delta^{4}\left(x^{\rho}-\right.\right.$ $\left.\left.x_{1}{ }^{\rho}\right) \mathrm{m} 1 \cdot u^{\mu} \cdot u^{v}+\delta^{4}\left(x^{\rho}-x_{2}{ }^{\rho}\right) \quad \mathrm{m} 2 \cdot u^{\mu} u^{v}\right]$ is the energy momentum tensor (EM) of two stars masses (in our case the system PSR $1913+16^{2}$ ) and $R_{\mu v \rho \sigma}$ the curvature Riemann tensor, the slash standing for contravariant derivative.

We can well distinguish in eq. (1) two terms whose the first, bound recursively to LHS $K_{\mu v}=1 / 2 \mathrm{c}\left(\partial_{\tau} g_{\mu v}\right)$, the deformation of metric with respect to proper time, proved at a great extent pre-eminent over the second one, (that is the double curl of the skew-symmetric tensor we called $C$ Riemann tensor $C_{\mu v}$ ) by tens of order of magnitude both for the former (see [1]) as for the latter solution by successive approximations, even though it has been evidenced that the contribution of the first term dropped in

2 We recall some hallmarks of the B1913+16 binary system: $\mathrm{m} 1: 1.4414(2) \mathrm{M}_{\text {sun }} ; \mathrm{m} 2: 1.3867(2) \mathrm{M}_{\text {sun }} ; e: 0.617$; orbital period $T: 7.72$ hours; major semi-axis a: 1.950E9 m; distance: 5kpc (16300 LY); dT/dt: -2.4056(51)E-12 (see also ATNF catalogue) 
a clearly way in this second approximation. The reader is strongly recommended to go through the two papers $[1,6]$.

\section{Methods}

Reviewing the antecedents in the previous calculus, as reported in [1], we chose the Minkowski metric as start metric in the successive approximations procedure, i.e. a constant cartesian metric $\left.g_{\mu v}\right|_{0}=\eta_{\mu v}$. But already to the second approximation in which the new metric is taken out integrating numerically with respect to the proper time

$$
\left.g_{\mu \nu}\right|_{1}=\eta_{\mu v}+2 c \int_{\tau_{0}}^{\tau} K_{\mu v}\left(x^{\rho} / \tau^{\prime}\right) d \tau^{\prime}
$$

the calculus proved incredibly CPU-consuming: the chief term in Eq.(1) yields elements of the deformation tensor as large as 125 Gbytes each, unmanageable for any computing software. The choice has been thereby to simplify as much as possible to getting the $2^{\text {nd }}$ order solution renouncing to covariant derivatives in the new metric concerning the term including the double curl (the term $C_{\mu v / / \sigma}{ }^{v}=-1 / 2 \operatorname{curl}\left(\varepsilon^{\delta \gamma \kappa \lambda} C_{\lambda \sigma / \kappa}\right.$ ) in Eq.(1)). Worth of noting the first term in Eq.(1), encompassing the curvature tensor $R_{\sigma \alpha}{ }^{\beta}{ }_{\mu}$ is therefore really a crucial one and together the most burdensome needing hundreds of GBytes. Thus some kind of ansatz is required that should be chosen with insight.

Because of the way which is has been got the $C$ tensor (see footnote 1) we have attempted to value the curvature tensor ${ }^{(1)} R_{\mu v \rho \sigma}=\left(\epsilon_{\mu v} / 4\right) C_{\rho \sigma}$ as to first approximation. Once evaluated the new $\left.g_{\mu v}\right|_{1}$ through integration of $K_{\mu \sigma}=$ $y_{\mu \sigma} \propto k / S$ according to Eq.(2), as done in the paper [1], we held at this stage the new Riemann tensor not to depend explicitly on the factors $k$ nor on $S$ because of the Christoffel symbols that it is composed of, which involves terms of the form $\left(g^{\sigma \mu} \partial_{\alpha} g_{\beta \sigma}-\ldots\right)$. However this choice is only of little interest in expressing the mean energy loss through the semi-axis and the simple $1 / S^{2}$ dependence, so an efficacious value, but it makes absolutely unreliable the physical estimate of the constant $S$ as already seen in [1] of order of $10^{14} \mathrm{~m}^{-1}$, while a just simple model to the second order has given the more acceptable result of $S=0.3261 \mathrm{~m}^{-1}$ which has allowed already to attribute the graviton the mass $10^{-43} \mathrm{Kg}$. Therefore, in effect we should expect some hinging on the ratio $k / S$. Taking as working hypothesis for the new Riemann tensor the more appealing makeshift expanding $\left(g^{\sigma \mu} \partial_{\alpha} g_{\beta \sigma}-\ldots\right)$ :

$$
{ }^{(2)} R_{\mu v \rho \sigma}=\frac{\epsilon \mu v}{4} \frac{k}{S}\left(\frac{3}{2 \pi^{2} r^{2}}\right) \partial_{\varphi}^{3} C_{\rho \sigma}
$$

improves the estimate of $S$ to $3.4 \mathrm{E}-14 \mathrm{~m}^{-1}$ for the three binary at issue, a value not far from the outcome of the complete direct calculus, still approximated, that we are going to carry out. Even though the Riemann tensor is denoted by the apex (2) the solution is always considered at $1^{\text {st }}$ order because all the tensors are raised/lowered by the flat metric. We get for this model (see the whole power at http://1drv.ms/1NOnDU6 ; there "asse" stands for major semi-axis, "th" for colatitudes, "f" for orbital phase)

$$
\langle\dot{E}\rangle=\frac{2.970 \mathrm{E}-24}{S^{3}}+\frac{1.085 \mathrm{E}-29}{S^{4}}+\frac{4.723 \mathrm{E}-18}{S^{2}}=-
$$

7.891E24 J/s against -7.959E24 J/s for Einstein's and $\dot{T}^{V}=-2.423 \mathrm{E}-12$ to within $0.7 \%$ with the experimental one against [13,sect. 4.1.2]- as seen later too:

$$
\begin{aligned}
& \dot{T}^{\text {Einst }}=-\frac{192 \pi G^{5 / 3}}{5 c^{5}} m 1 \cdot m 2(m 1+m 2)^{-1 / 3}\left(\frac{T}{2 \pi}\right)^{-5 / 3} . \\
& \frac{1}{(1-e)^{7 / 2}}\left(1+\frac{73}{24} e^{2}+\frac{37}{96} e^{4}\right)=-2.40242(2) \mathrm{E}-12
\end{aligned}
$$

In the following we are referring to the model of Eq.(3) as 1st approximation Vortex Model. As checked with CILEA supercomputer section the direct calculus of energy loss via equ.(1) is very beyond standard RAM performance (see footnote 3 ) without taking in account the lack of manageability of the output files to average in turn. The strategy that has been adopted here therefore was to guess that the passage of integration of Eq.(2) results in a staircase-like behaviour for each of the components of $F_{\mu v}[\tau(\varphi)] \quad 2 c \int_{\tau_{0}}^{\tau} K_{\mu \nu}\left(x^{\rho} / \tau^{\prime}\right) d \tau^{\prime}=\int_{0}^{\varphi^{\prime}} F_{\mu \nu}(\tau(\varphi)) d \varphi$ that can be well interpolated by the straight line of the kind $F_{\mu v}(\varphi=1.37) \cdot \varphi, \varphi$ being the orbital phase.
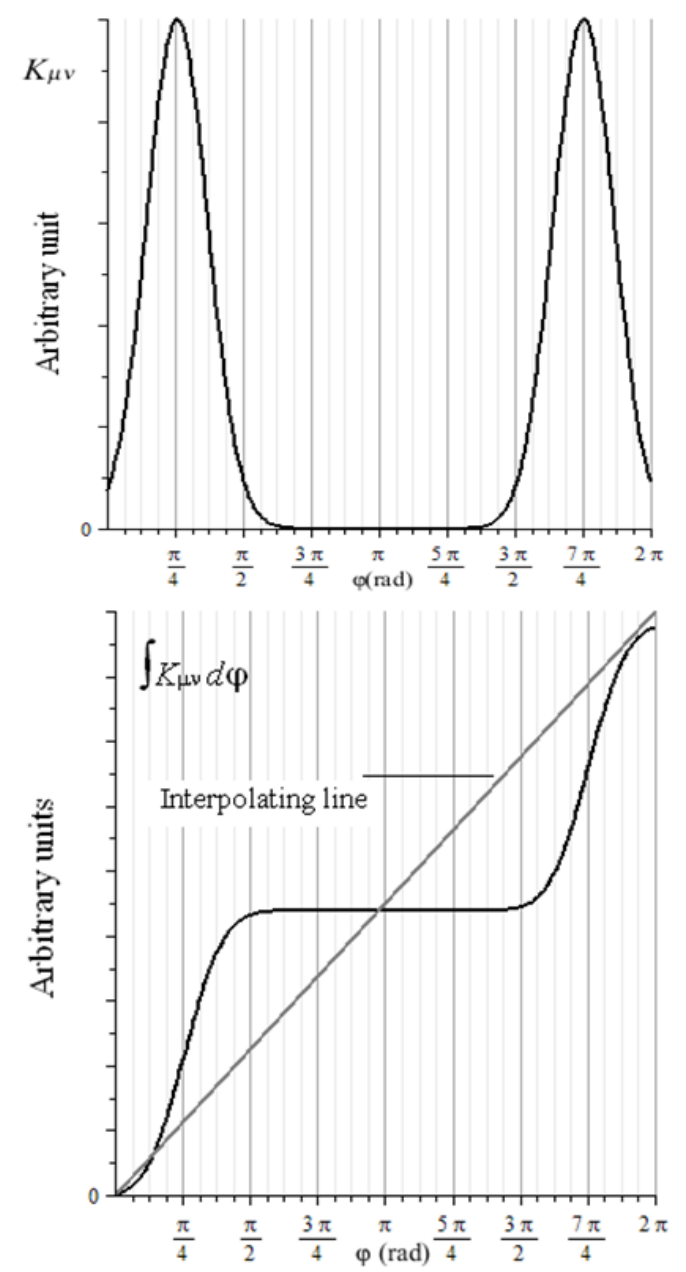

Figure 1. Integration method used in Eq.(2) for working out the new metric $\left.g_{\mu \nu}\right|_{1}$ and hence its inverse. The integrand containing $K_{\mu \nu}$ is patterned by two Gaussian functions as fitted by B1913+16 outcomes . The right interpolation is found for a slope line slope at the value of integrand function $F_{\mu \nu}[\tau(\varphi)]$ corresponding to $\varphi=1.37$ 
This fetch is put on to obtain $\left.g^{\mu v}\right|_{1}$ while for $\left.g_{\mu v}\right|_{1}$ a 3 steps Simpson algorithm has been adopted. Moreover, taking $=\boldsymbol{n}=(\Theta, \Phi)$ the direction of propagation of the wave from coordinate system of the orbit plane, as can be verified heuristically on data also from [1] concerning $\mathrm{B} 1913+16$, it is a good approximation to put $d \bar{E} / d t d \Theta \mid\left(\Theta=\Theta^{*}, \varphi=\varphi_{\text {peak }}\right) \approx<d E / d t>_{T}$, especially to reduce the burden of calculation (especially introduced by the inverse $\left.g^{\mu v}\right|_{1}$ ). This may be justified by seeing that the distribution function may be roughly shaped as two triangles each centered about a unit interval on $\varphi$. Then, since it is noted the differential energy loss not to depend significantly on $\Theta$, the colatitude $\Theta^{*}$ has been fixed to $\pi / 2$ (edge-on line of sight). We have over-marked $E$ because the power should be indeed averaged over time, so a factor to multiply $\Omega_{0} / \dot{\varphi}$ is needed to turn back the average on the orbital anomaly, namely

$$
\begin{aligned}
& \frac{1}{T} \int_{0}^{T} d t \int_{0}^{2 \pi} \frac{d E}{d t d \Omega} d \Phi=\frac{\Omega_{0}}{2 \pi} \int_{0}^{2 \pi} \frac{1}{\dot{\varphi}} d \varphi \int_{0}^{2 \pi} \frac{d E}{d t d \Omega} d \Phi= \\
& \int_{0}^{2 \pi} d \varphi \frac{d \bar{E}}{d t \sin \Theta d \Theta}
\end{aligned}
$$

with $\Omega_{0}=2 \pi / T$ and

$$
\dot{\varphi}=\left[G(m 1+m 2) a\left(1-e^{2}\right)\right]^{1 / 2} / \rho^{2}(\varphi) .
$$

The $2 \pi$ term dividing in Eq.(5) is omitted however in that the integration over the anomaly $\Phi$ cancels it because the asymptotic metric to manage the strength tensor of Vortex theory (see later) is the usual Minkowski's which does not depend on $\Phi$. Thus in the following plots for $d E / d t d \Omega$ it is meant the integrand of Eq.(5) that we could style as "effective" differential power. Besides, the wrinkle has been preferred to focus the reckoning in correspondence with each one of the two main resonance peaks wherein to fit the two values of $S$. Actually, if one proceeded the whole calculus with the value of $S$ sought for the $1^{\text {st }}$ peak, the second one on the right would be found unequal of several orders, just because of the very rough but necessary simplification in getting the new metric in Eq.(2) through the numeric integral (as said, only one step per orbital interval in the azimuthal variable ${ }^{3}$ ) of deformation tensor carried out in such an approximation, inasmuch as the new metric becomes less accurate as the interval of integration extends over the whole $2 \pi$ angular orbital journey, so occasioning a greater growth in systematic errors. For this purpose the interval $[0,2 \pi]$ has been broken in the two $[0, \pi]$ and $[\pi, 2 \pi]$ in each of which has been chosen to calculate by Maple the effective power function $d E / d t d \Omega$ with respect to the anomaly in steps of $0.1-0,2 \mathrm{rad}$ in the orbital plane. In short, this trick saves from a awkward integral over $\Theta$. Worth to note is the fact that this does not imply loss of generality in that is seen

3 We know from the sampling theorem that in order to rebuild a periodic function, there is need for a sampling frequency at least double of that maximum of its spectrum (Nyquist frequency). Nevertheless taking yet three step per interval entailing a course estimate of the numerical integration need about 500Gbytes, while four steps per interval would involve too much bigger files to manage for $K_{\mu v}$, of order of Terabytes. that in Vortex Theory there is not any uncoupling of the $\oplus$ from the $\otimes$ mode in the orbital plane line of sight as in TGR (see probl.3.2 in [13]) although the $\otimes$ mode here is differently defined. But let us just recall now the main steps to get the expression for $d E / d t$. We have applied firstly this method to the classical B1913+16 binary system while comparing the results with the other more recent B1534+12 [17], J0737-3039 [16] and B2127+11C[19] discovered in 1991, 2003 and 1989. In carrying out the strength tensor $\Phi_{\mu v \sigma}$ (see Appendix B and [6]) for the energy loss calculus ${ }^{4} d E / d t=c \int t_{s}^{0} n^{s} r^{2} d \Omega$, with $t_{s}^{0}=-\Phi_{s}{ }^{\sigma \alpha} \Phi_{\sigma \alpha}^{0}$, the azimuthal variable has to be taken as a function of delayed time and so also of distance from the observer, i.e. $\varphi \equiv \varphi(t-r / c)$, which raises of a great deal the burden of calculus. This has led to a function of $S$ $f(S)$ to be inserted in the differential relation of third Kepler's law outputting a polynomial equation of which to find the root:

$$
\begin{aligned}
& \equiv 2 T<\frac{d T}{d t}>-\frac{4 \pi^{2}}{G(m 1+m 2)} 3 a^{2}\left(<\frac{d E}{d t}>\frac{2<r>^{2}}{G m 1 m 2}\right) \\
& =0
\end{aligned}
$$

Fortunately, the function $f(S)$, being monotonic, has been able to be tackled through the dichotomic method in figuring out the root (other methods as secants or Newton'one would introduce propagation errors).

\section{Results}

\begin{tabular}{|c|c|c|c|}
\hline \multicolumn{4}{|l|}{ ere } \\
\hline & $S_{1 \text { st peak }}\left(\mathrm{m}^{-1}\right)$ & $S_{2 \text { nd peak }}\left(\mathrm{m}^{-1}\right)$ & $\begin{array}{c}S=\sqrt{S_{1} S_{2}} \\
\left(\mathrm{~m}^{-1}\right)\end{array}$ \\
\hline B1913 +16 & $\left.S\right|_{\varphi=0.4}=5.293 \mathrm{E}-19$ & $\left.S\right|_{\varphi=5.6}=1.979 \mathrm{E}-19$ & $3.236 \mathrm{E}-19$ \\
\hline J0737-3039 & $\left.S\right|_{\varphi=0.4}=1.122 \mathrm{E}-19$ & $\left.S\right|_{\varphi=5.31}=8.442 \mathrm{E}-20$ & $9.732 \mathrm{E}-20$ \\
\hline B1534 +12 & $\left.S\right|_{\varphi=1.11}=3.473 \mathrm{E}-19$ & $\left.S\right|_{\varphi=5.31}=1.131 \mathrm{E}-18$ & $6.266 \mathrm{E}-19$ \\
\hline \multirow[t]{3}{*}{$\mathrm{B} 2127+11 \mathrm{C}$} & $\left.S\right|_{\varphi=0.5}=3.302 \mathrm{E}-19$ & $\left.S\right|_{\varphi=5.6}=1.062 \mathrm{E}-19$ & $1.873 \mathrm{E}-19$ \\
\hline & & $\begin{array}{c}S=\left(S_{a} S_{b} S_{c} S_{d}\right)^{1 / 4} \\
\rightarrow\end{array}$ & $2.466 \mathrm{E}-19$ \\
\hline & $\begin{array}{c}d E / d t d \Omega(\mathrm{J} / \mathrm{s}) 1 \mathrm{st} \\
\text { peak }\end{array}$ & $\begin{array}{c}d E / d t d \Omega(\mathrm{J} / \mathrm{s}) \\
\text { peak }\end{array}$ & \\
\hline B1913 +16 & $\left.\right|_{\varphi=0.4}=7.873 \mathrm{E} 24$ & $\left.\right|_{\varphi=5.6}=7.873 \mathrm{E} 24$ & \\
\hline J0737-3039 & $\left.\right|_{\varphi=0.4}=2.394 \mathrm{E} 24$ & $\left.\right|_{\varphi=5.31}=2.393 \mathrm{E} 25$ & \\
\hline B1534 +12 & $\left.\right|_{\varphi=1.11}=3.338 \mathrm{E} 23$ & $\left.\right|_{\varphi=5.31}=3.338 \mathrm{E} 23$ & \\
\hline $\begin{array}{c}\mathrm{B} 2127 \\
+11 \mathrm{C}\end{array}$ & $\left.\right|_{\varphi=0.5}=1.140 \mathrm{E} 25$ & $\left.\right|_{\varphi=5.6}=1.140 \mathrm{E} 25$ & \\
\hline
\end{tabular}

The reckoning has yielded the following double $S$ constant series in the two intervals:

Table 1. Fitted $S$ constant and effective differential power values at the resonances

Taking the geometric average gives for $S=2.47 \mathrm{E}-19 \mathrm{~m}^{-1}$. Taking also the semi-dispersion for error estimate about

4 as known from the free divergence of EM tensor. Indeed, we should strictly have written $d E / d t=c \int t_{s}^{0} n^{s}(L(g))^{1 / 2} d \sigma$ with $L(g)$ the restriction of the metric over a surface enclosing the binary system, but we take it instead as the usual jacobian in spherical coordinates in account of the sidereal distance from it. 
each binary system, the relative error may be expressed as $\Delta \log \left(S_{a} S_{b} S_{c} S_{d}\right)^{1 / 4}$ getting for $\Delta S= \pm 1.16 \mathrm{E}-19 \mathrm{~m}^{-1}$.
With these value we are able now to draw the energy loss with respect to the orbital phase.
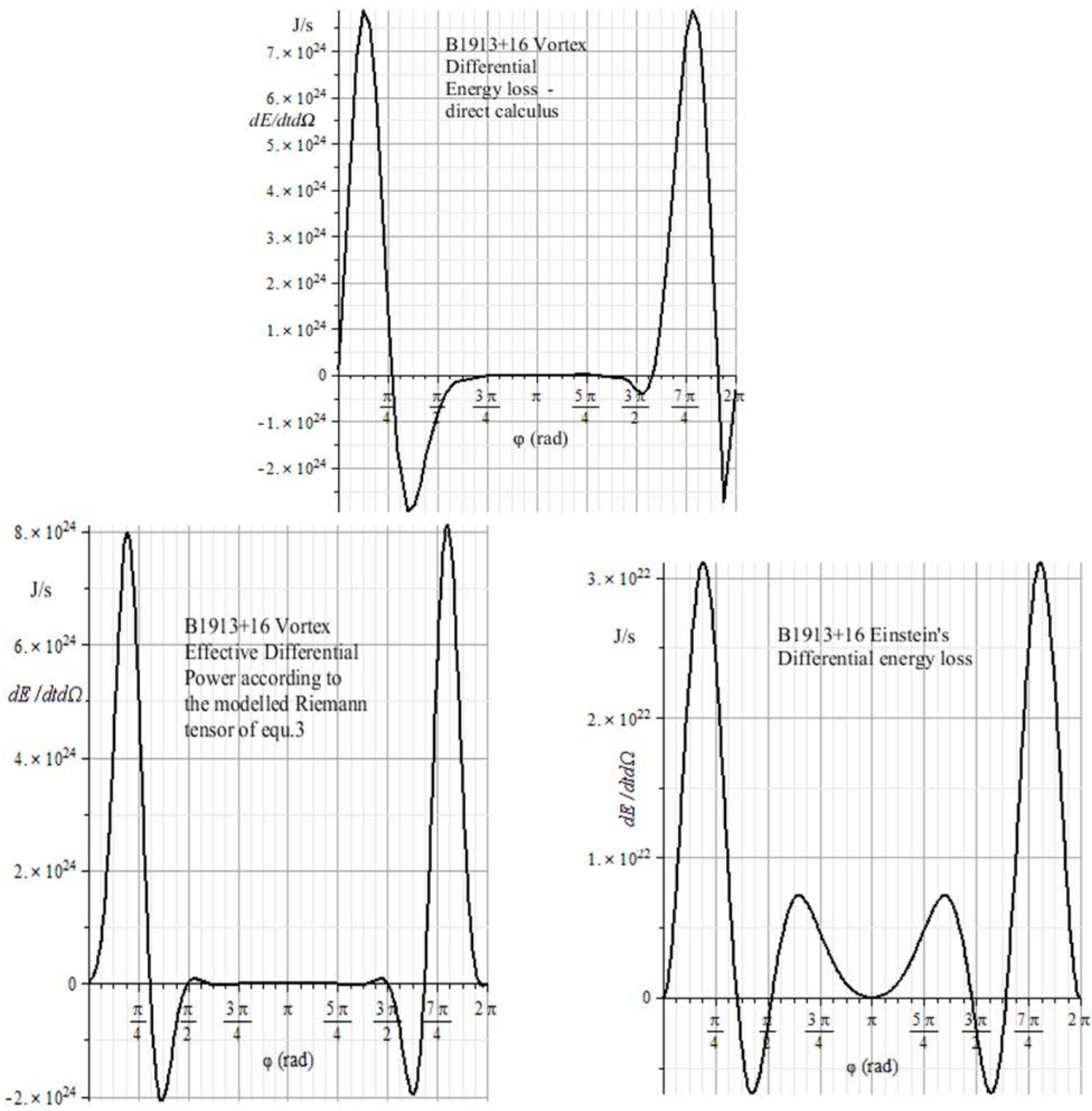

Figure 2. Plot of $2 \pi$ periodic emitted effective instantaneous power for B1913+16 in the Vortex model with the two-fold peak (whose number depends chiefly on $e$ ) to $1^{\text {st }}$ approximation and in the direct calculus in comparison with Einstein's one at polar angle $\theta=\pi / 2$ and azimuth $\Phi=\pi / 2$. The broken line in the first figure joins the various calculated points. Comments on differents orders of magnitude will be given further on

We report the instantaneous emitted power for a given direction at polar angle $\theta=\pi / 2$ and azimuth $\Phi=\pi / 2$ corrected of the time averaging factor $\Omega_{0} / \dot{\varphi}$ in the direct calculus versus first approximation (Figure 2) for the Vortex Model for the B1913+16 system. As a collation, we report also the Einstein's one as known proportional to the products of third derivatives of the quadrupole moment [9]:

$$
\begin{aligned}
& \left.W\right|_{\Omega} ^{\text {Einatein }}=\frac{1}{9}\left(\frac{G}{4 \pi c^{5}}\right)\left(\frac{1}{2} \frac{\partial^{3} Q^{r s}}{\partial \tau^{3}} \frac{\partial^{3} Q^{r s}}{\partial \tau^{3}}\right. \\
& -\frac{\partial^{3} Q^{l s}}{\partial \tau^{3}} \frac{\partial^{3} Q^{l m}}{\partial \tau^{3}} n^{s} n^{m} \\
& \left.+\frac{1}{4} \frac{\partial^{3} Q^{l r}}{\partial \tau^{3}} \frac{\partial^{3} Q^{s m}}{\partial \tau^{3}} n^{l} n^{r} n^{s} n^{m}\right) \sin \Theta d \Phi d \Theta \\
& n^{s}=x^{s} / r \quad Q^{r s}=\int\left(3 x^{\prime r} x^{\prime r}-r^{\prime 2} \delta_{r}^{s}\right) D\left(x^{\prime}\right) d^{3} x^{\prime}
\end{aligned}
$$

It is worth mentioning that a single point in the direct calculus takes about three hours of processing. We note in this direct calculus a moving to the right of the secondary negative peak but we have had to cope with a larger reckoning approximation, although more realistic, with respect to an ad hoc model such as that of Eq.(3). In the direct calculus the peaks are obtained correspondently to the values of Table 1.

Our strict criterion in calculus of precision, as recalled in [1], is given by the effective symmetry of the deformation tensor (Figure3(a)) and the skew-symmetry of $C$ Riemann tensor (Figure 3(b)) along the orbit, i.e. to the semi-axis extent concerning the two tensors that appear in the evaluation of the integral in Eq.(1), as included in the constraint on the variables of the Dirac delta of the EM tensor of the two point masses of the binary system. The accuracy is shown bettered with respect to our model Eq.(3). 

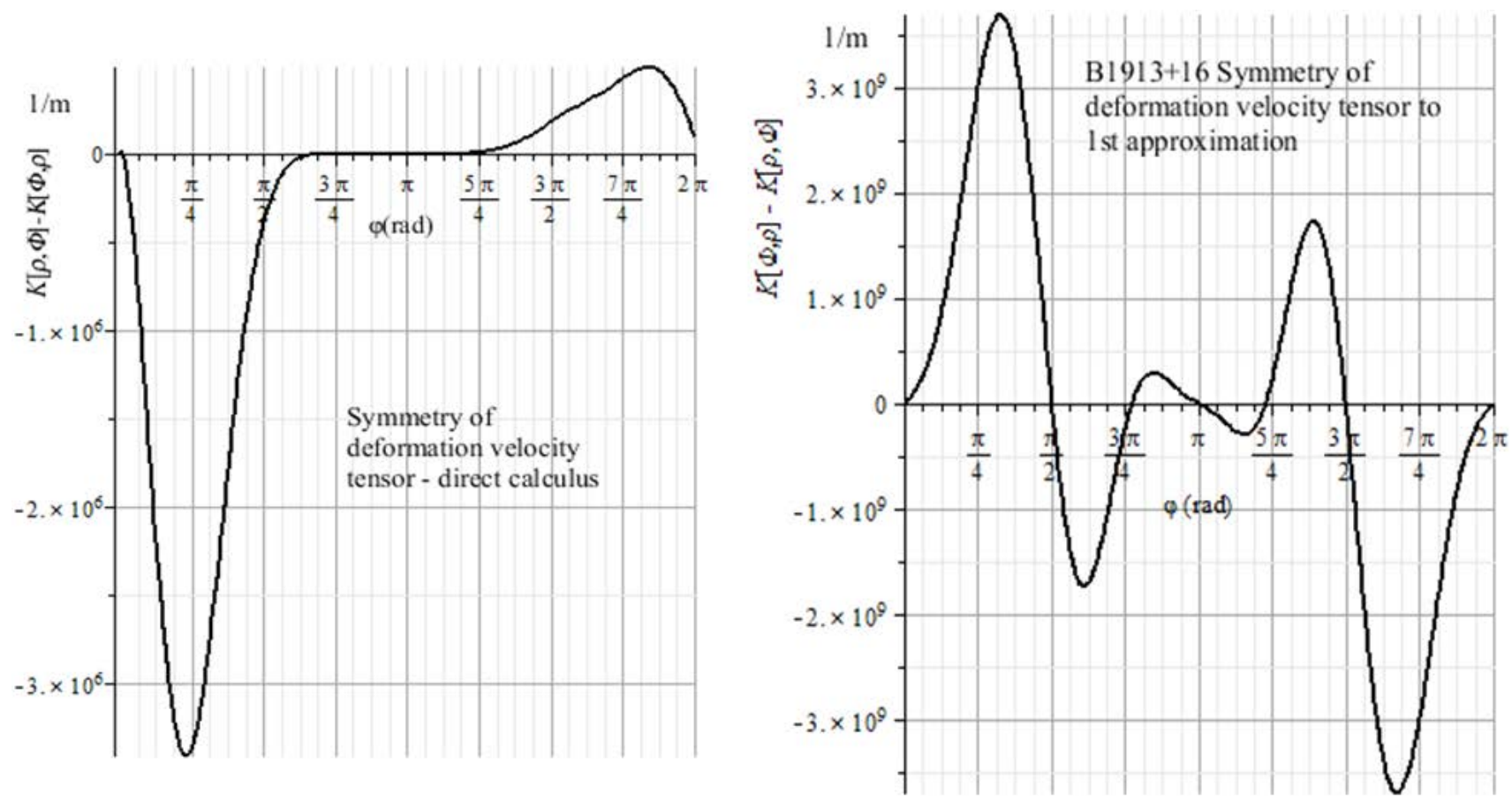

Figure 3. (a): Plot of $2 \pi$ periodic $K_{24}-K_{42}$ representative of deformation speed precision in polar coordinates in the first two steps iterative solution for B1913+16, in function of the azimuthal angle along the elliptic orbit
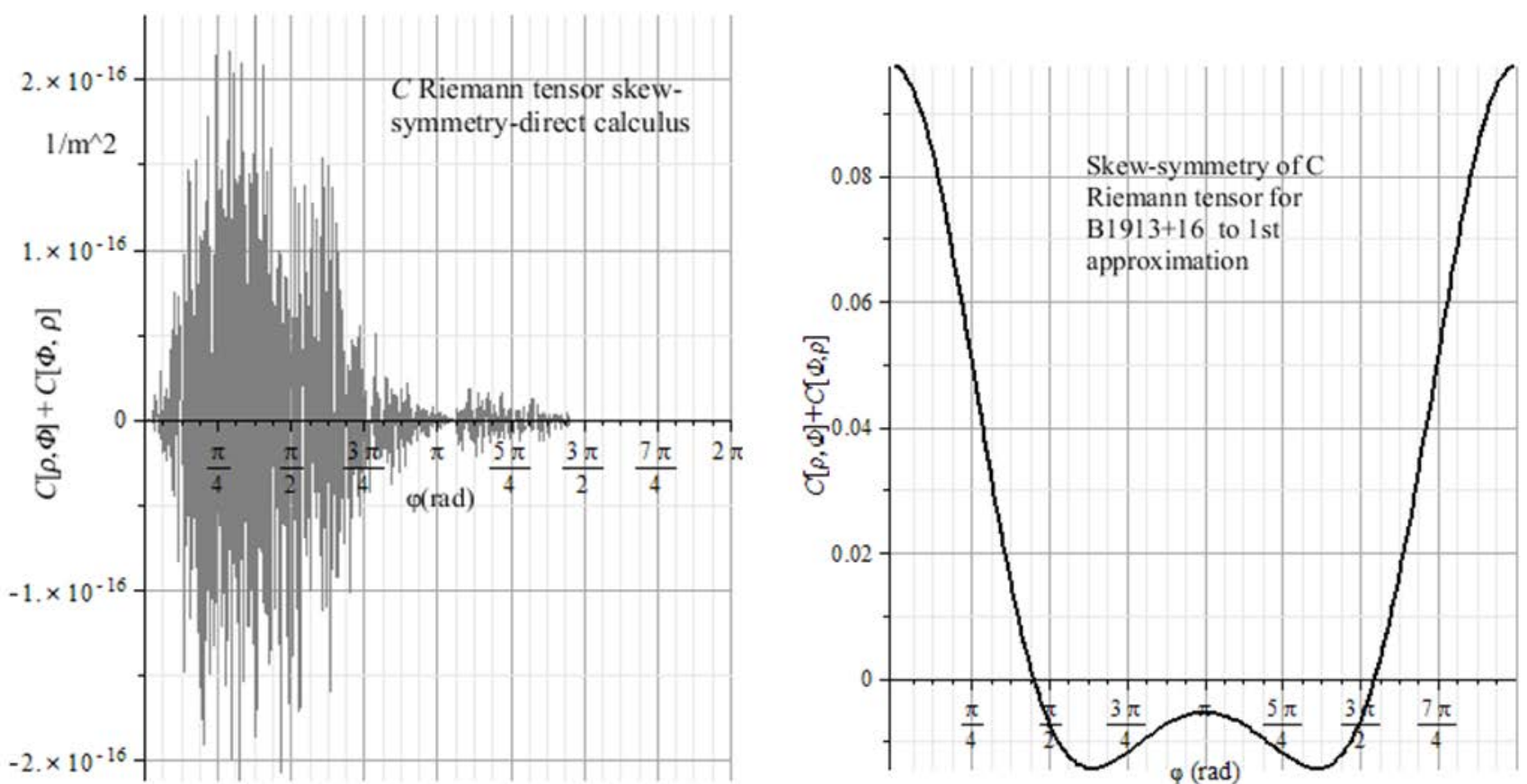

Figure 3(b). Plot of $2 \pi$ periodic $C_{24}+C_{42}$ representative of skew- symmetric Riemann tensor precision in polar coordinates in the first two steps iterative solution for B1913+16, in function of the azimuthal angle along the elliptic orbit

We then report the B1913+16 orbital period decay advancing at periastron time in Figure 4. Expressing it ${ }^{5}$ [13] as $t_{n}-n T=\frac{\dot{T}}{2 T} t_{n}^{2}$ at the modified julian epoch $t_{n}$ (MJD), we may collate directly the Vortex Model with TGR tendency for $\dot{T}$ in the matching of the experimental

$5 \quad$ The formula is got by expressing the accumulated phase as function of time $\phi(T)=2 \pi \int_{0}^{T} v(t) d t$, expanding the frequency $v$ to 2nd order and drawing out the difference between the times of passage at periastron and the n-th proper period time $n / v_{0}$ fitted curve. We remember The TGR prediction is to within the celebrated 0.2 percent [14].

By inserting the mean energy as from the virial theorem in the 3th Kepler's law and differentiating, the variation of the orbital period comes out as $\dot{T}=-\frac{3}{2} \frac{\langle\dot{E}\rangle}{\langle E\rangle} T$, $\langle E\rangle=-\frac{G \cdot m_{1} \cdot m_{2}}{2 \cdot a}$; By replacing the values, one gets $\dot{T}=$ -2.4042(2)E-12 for TGR versus -2.417E-12 for the Vortex Model against $\dot{T}^{\text {Exp }}=-2.4056(51) \mathrm{E}-12$ [14]. We so have 
$0.13 \pm 0.21 \%$ agreement for TGR against $0.5 \%$ for Vortex.

However better can be found for the other two systems.
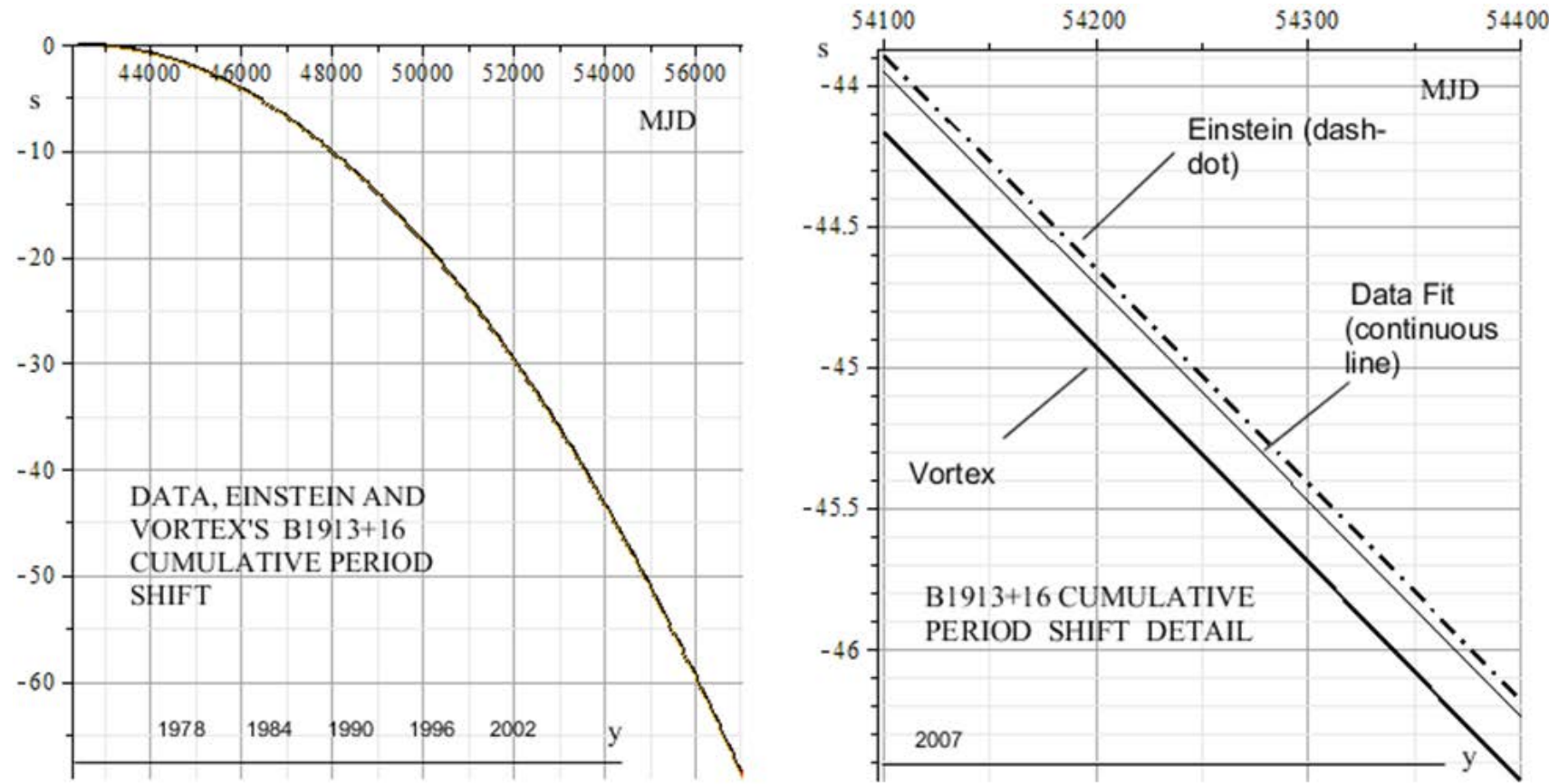

Figure 4. Period change in the damping of B1913+16 system with respect to the fitted data versus Modified Julian Day Time from 1975 to 2013 . We can observe how the TGR approximates closer up to $0.2 \%$ to the expected than Vortex Theory prediction which indeed is seen in the detail inset to anticipate of about $0.5 \%$

For what concerns the other star two systems B1534 and the double pulsar J0737 we too report as before the differential energy loss in the Vortex direct calculus and $1^{\text {st }}$ approximation paralleled with the respective Einstein's prevision. The differential functions prepared to be averaged by the usual factor $\Omega_{0} / \dot{\varphi}$ along the orbit appear to disagree of at least two order of magnitude as to B1534 and J0737 as well as for the other two systems as seen below. However what physically matters is the integral over the solid angle to get $\langle d E / d t>$ and this points out all the more we are dealing with two obviously different models. Vortex Theory is seen to be more reliable in predicting period shift as for these two systems. Data values other than available are extrapolated assuming as before the rotator model of the accumulated phase in the relative motion $\frac{1}{2 \pi} \phi(T)=v T+\frac{1}{2} \dot{v} T^{2}+\ldots$.

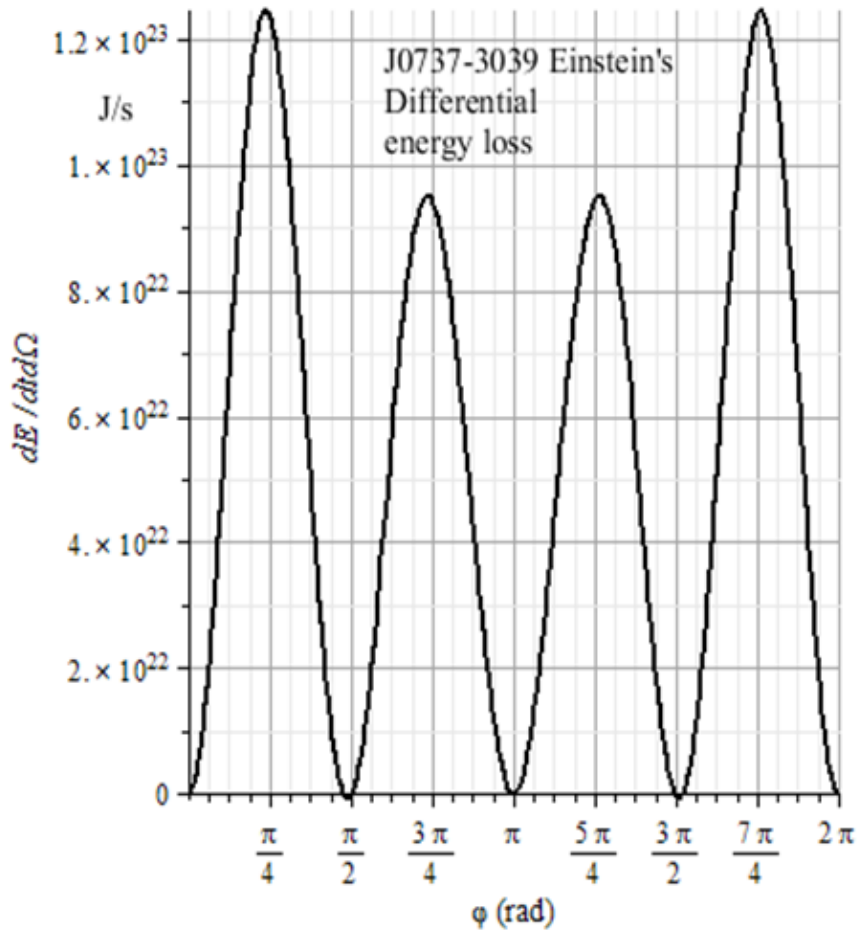

Figure 5(a). J0737+39 effective differential energy loss for Vortex and Einstein models respectively to a given direction at polar angle $\theta=\pi / 2$ and azimuth $\Phi=\pi / 2$. The system is at a distance of $1900 \mathrm{LY}$ and has eccentricity 0.088 with major semi-axis of $8.804 \mathrm{E} 8 \mathrm{~m}$ 

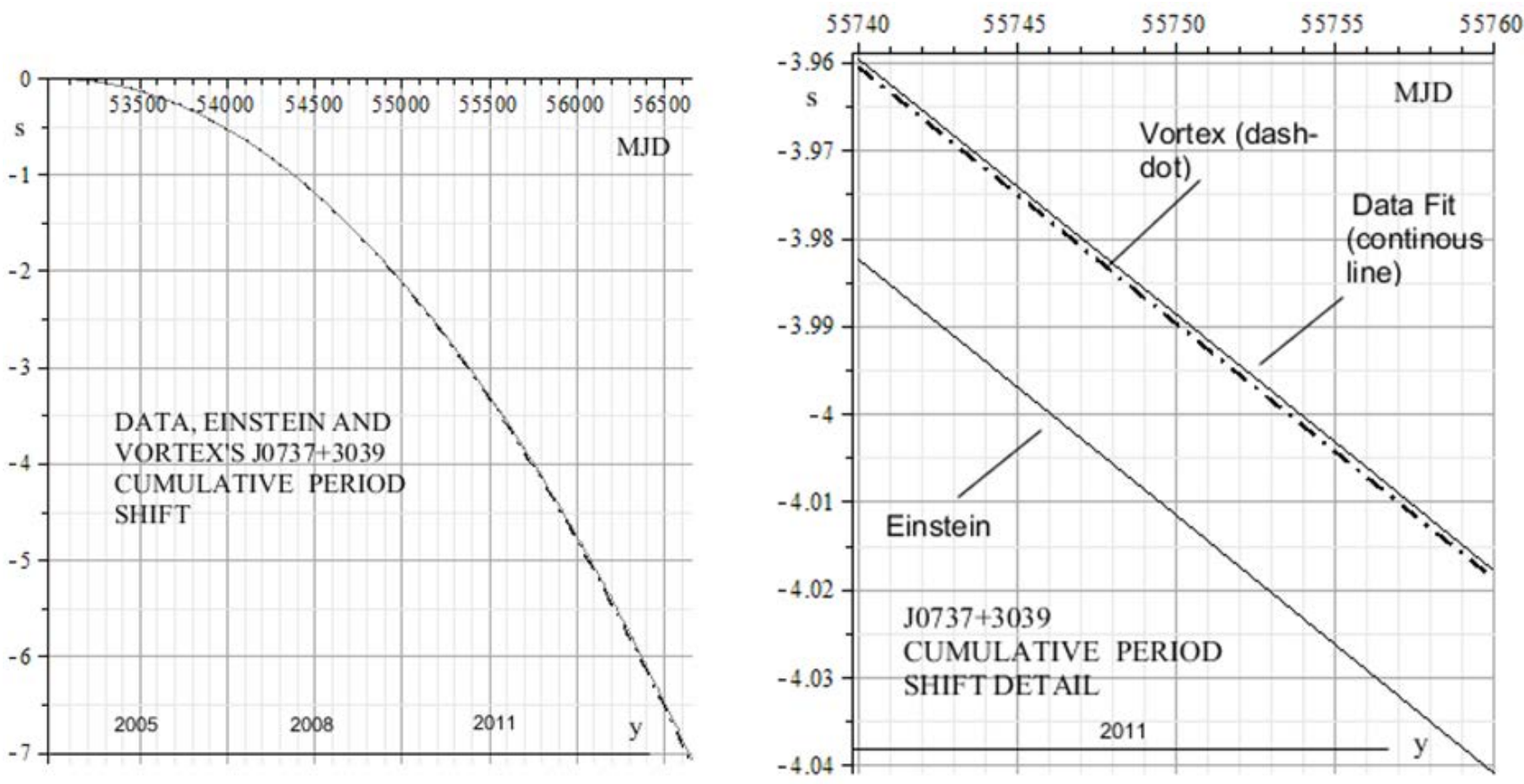

Figure 5(b). Period change in the damping of J0737 system with respect to the fitted data versus Modified Julian Day Time from 2003 to 2013. We can observe how Vortex Theory prediction can barely be discriminated to within the approximation precision, while TGR is seen in the detail inset to anticipate of about $0.6 \%$ the data fit delay

About the relativistic system J0737 we calculated $\dot{T}=$ 1.2523E-12 for Vortex vs $\dot{T}=-1.2592 \mathrm{E}-12$ for Einstein's theory against the intrinsic experimental value $\dot{T}^{E X P}=$ -

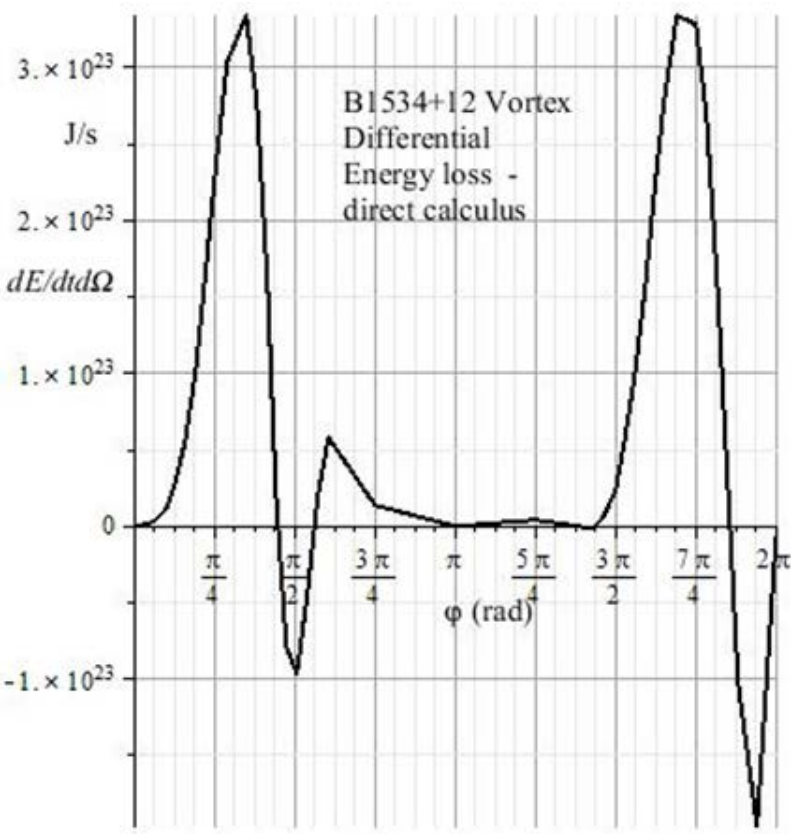

1.252(12)E-12 [16]. Hence the most precise test ever obtained for Vortex's Model.

Figure 6. (a). Differential effective energy loss of B1534 system for Vortex and Einstein models respectively to a given direction at polar angle $\theta=$ $\pi / 2$ and azimuth $\Phi=\pi / 2$. The system is at a distance of $33000 \mathrm{LY}$ and has eccentricity 0.681 with major semi-axis of $1.97 \mathrm{E} 9 \mathrm{~m}$

We may again observe in Figure6(b) the overcoming of Vortex theory to TGR. The outcome $\dot{T}^{V}=-0.173 \mathrm{E}-12$ seems indeed to resolve the small discrepancy puzzle between TGR and observed value of period variation, proper of this binary system. In comparison with $\dot{T}^{\text {Exp }}=$ $0.174(11) \mathrm{E}-12$ we have in fact $\dot{T}^{T G R}=-0.192 \mathrm{E}-12$ [17].

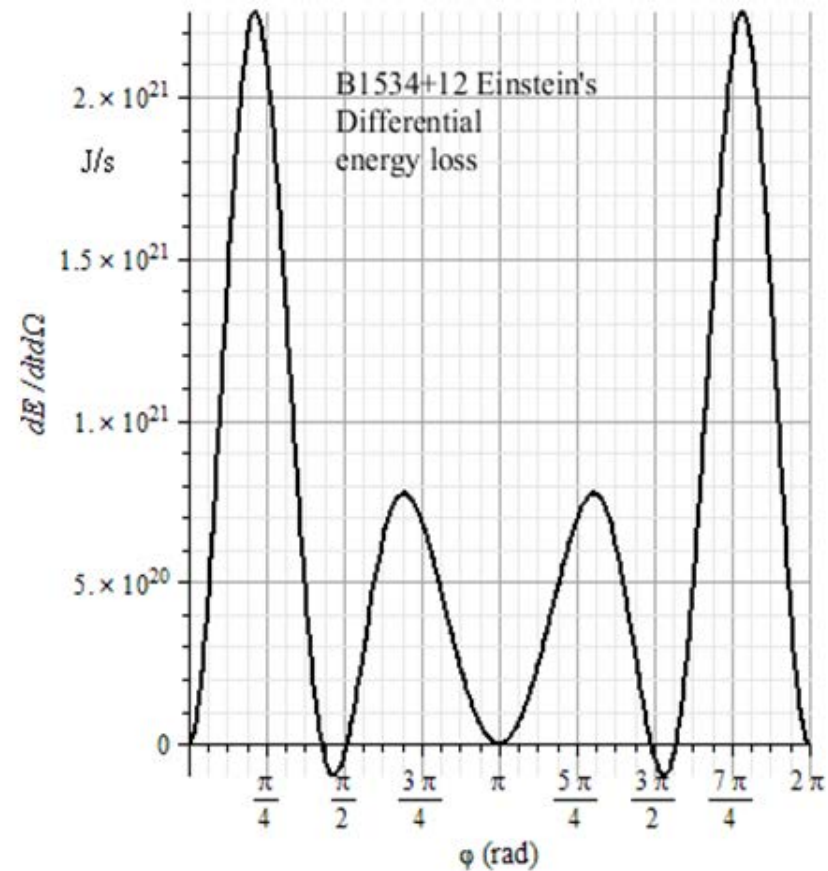

At last we have reviewed the B2127+C11 dwarf-NS system very similar to B1913+16 for which $\dot{T}^{V}=-3.98 \mathrm{E}-$ 12 suits to $\dot{T}^{\text {Exp }}=(-3.95 \pm 0.13) \mathrm{E}-12$ as well as $\dot{T}^{T G R}=-$ $3.98 \mathrm{E}-12$. However uncertainties prevent us to usefully collect directly the models. We report the angular distribution of the radiated energy against Einstein's one: 

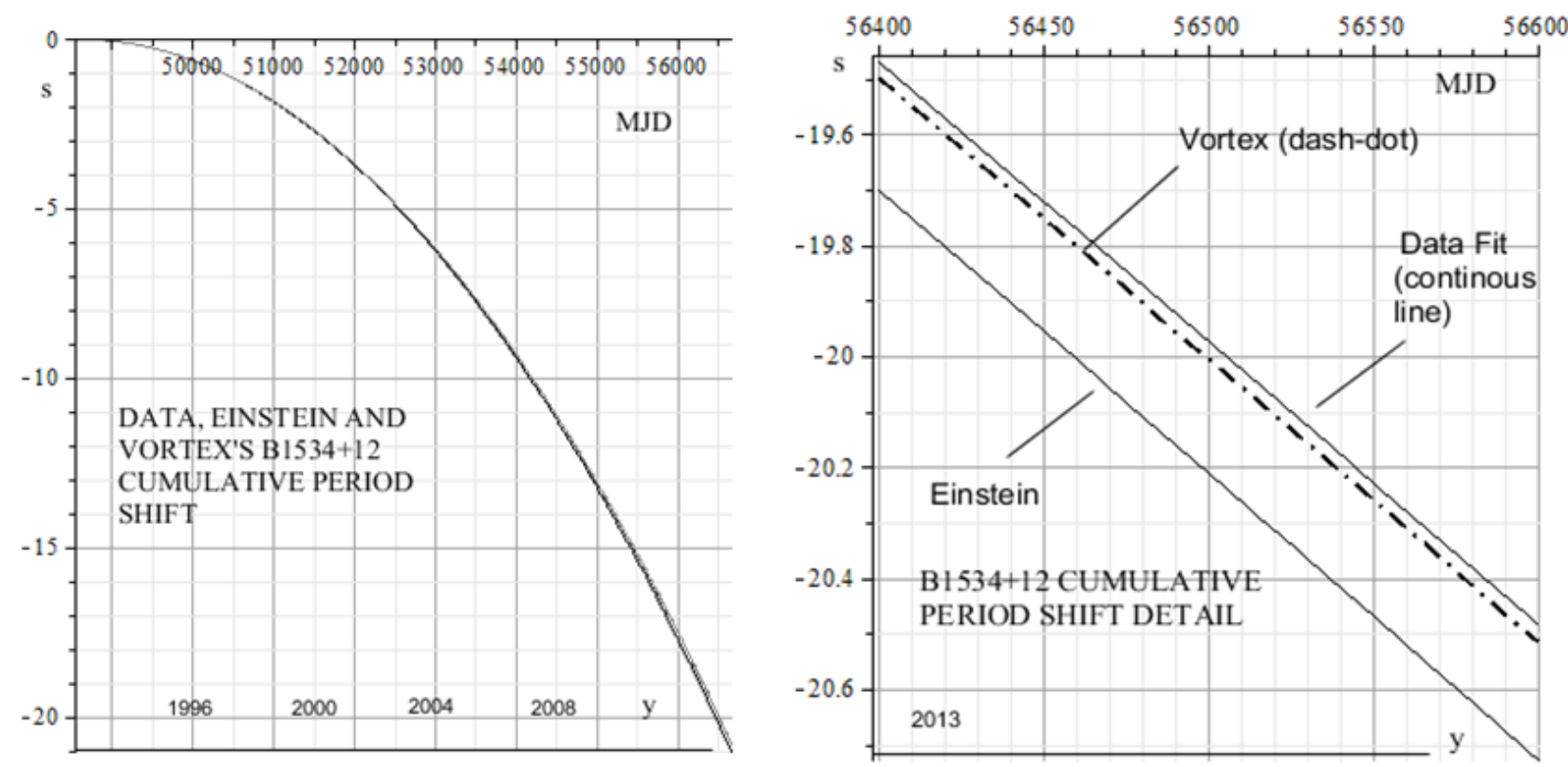

Figure 6.(b): Period change in the damping of B1534+12 system with respect to the fitted data versus Modified Julian Day Time from 1991 to 2013. We can observe how Vortex Theory approximates closer up to $0.2 \%$ to the expected data than TGR prediction which indeed is seen in the detail inset to anticipate of about $10 \%$
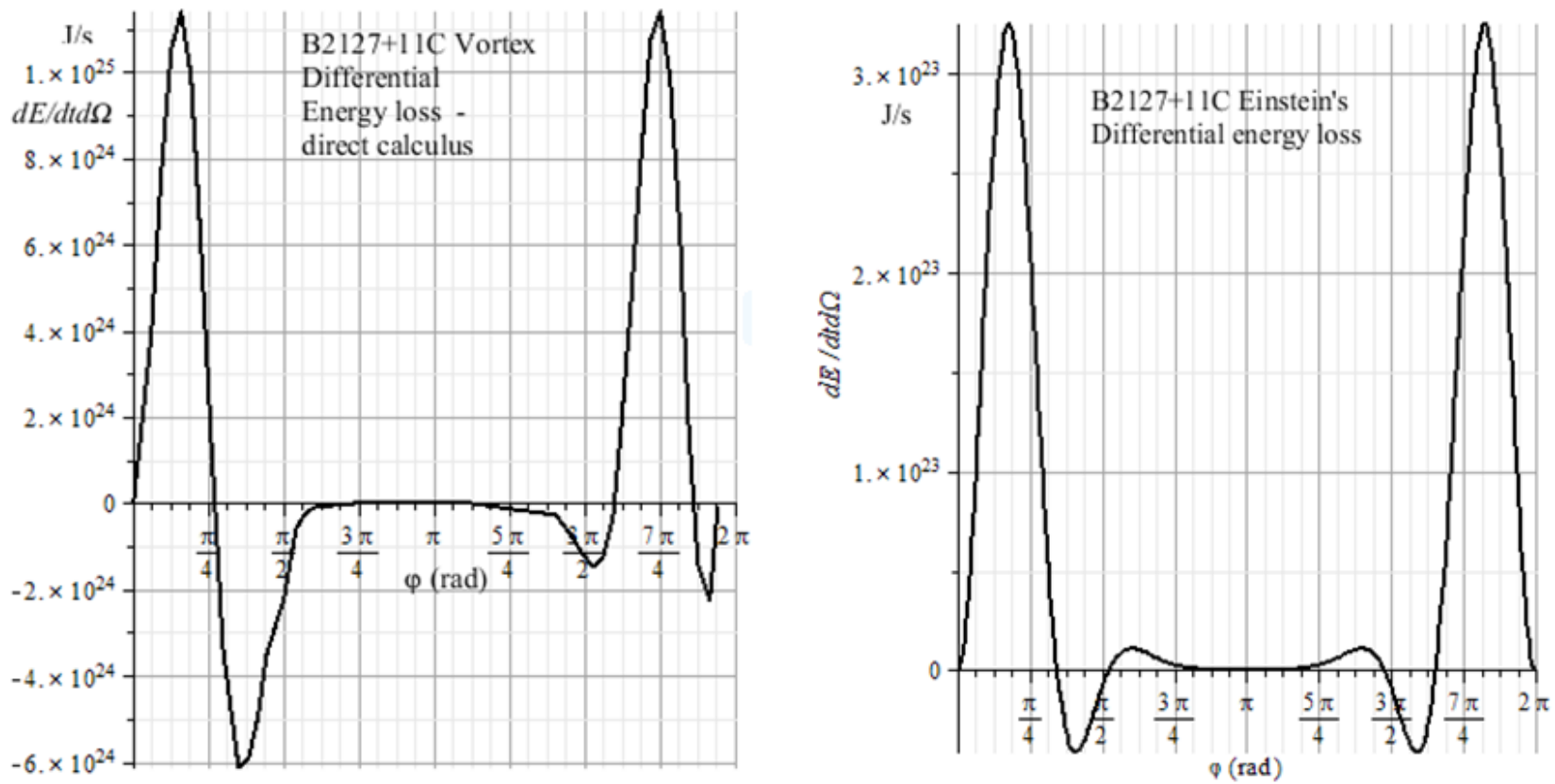

Figure 7. Effective differential energy loss of B2127+11C system for Vortex and Einstein models respectively to a given direction at polar angle $\theta=$ $\pi / 2$ and azimuth $\Phi=\pi / 2$. The system is at a distance of $2900 \mathrm{LY}$ and has eccentricity 0.274 with major semi-axis of $2.283 \mathrm{E} 9 \mathrm{~m}$

\section{Discussion and Conclusions}

In spite of all glitches of numerical quadratures, we deem the new value of the constant $S=(2.5 \pm 1.2) \mathrm{E}-19 \mathrm{~m}^{-1}$ more meaningful than our model at first approximation $\left(\left.S\right|_{1}=3 \mathrm{E}-14 \mathrm{~m}^{-1}\right)$. As has been said in the reference article [6], it can be related to the mean free path of graviton through a Compton-like wavelenght $=S^{-1}=h / m c$ meant to attribute a value to the "inertial mass" of graviton, in this case amounting to $(5.5 \pm 2.6) \mathrm{E}-61 \mathrm{Kg}$, not far from former bounds [7,8,11,12]. Applying this Vortex Model to keplerian problems at galactic distances as for galaxy rotational curves or solar system issues is a very entangled challenge to be faced with intensive amount of skill analysis. Further verifications and bearing out for consistency of this result other than bettering of statistics should also be provided from other binary NS-NS or dwarf/NS systems such as for instance J1756-2251 and J1518+4904. An indirect confirmation should come also from the energy radiated from the problem of Black Hole in-falling into another [15], excepting over dimensioned RAM required.

\section{Appendix A: Tidal deformations in Vortex Theory}

Because instructive how Vortex Model works, we want to get the strain in a given direction as expressed by the formula (17) of [1] caused by the tidal force of a 
gravitational wave impinging on a certain region freefalling in the space-time. We will follow the notations therein by leaning on the derivation from the quoted books of Ferrarese and Sokolnikoff $[4,10]$. Let us consider the evolution with respect to the proper time of a continuum of identifiable material particles from the reference or initial configuration $C_{0}$ to the actual one $C_{\tau}$, which we refer to the reference frames $\mathrm{X}$ and $\mathrm{Y}$ respectively, (they are different in general, see the two terns of Figure8), whose a typical point $\mathrm{P}$ will have coordinates $x^{\mu}$ and $y^{\mu}$ in passing from the undeformed state to the deformed of final one of the continuum. Thus, we may take the deformation of $C_{0}$ in $C_{\tau}$ to be a continuous and one-to-one function so that the transformation of the curvilinear coordinates $x^{\mu}$ of the material particle $P$ is singled-valued:

$$
y^{\mu}=y^{\mu}\left(x^{0}, x^{1}, x^{2}, x^{3} / \tau\right), \quad(\mu=0,1,2,3)
$$

depending on whether we use the initial or final coordinates as independent variables we will have the socalled eulerian or lagrangian motion standpoint.

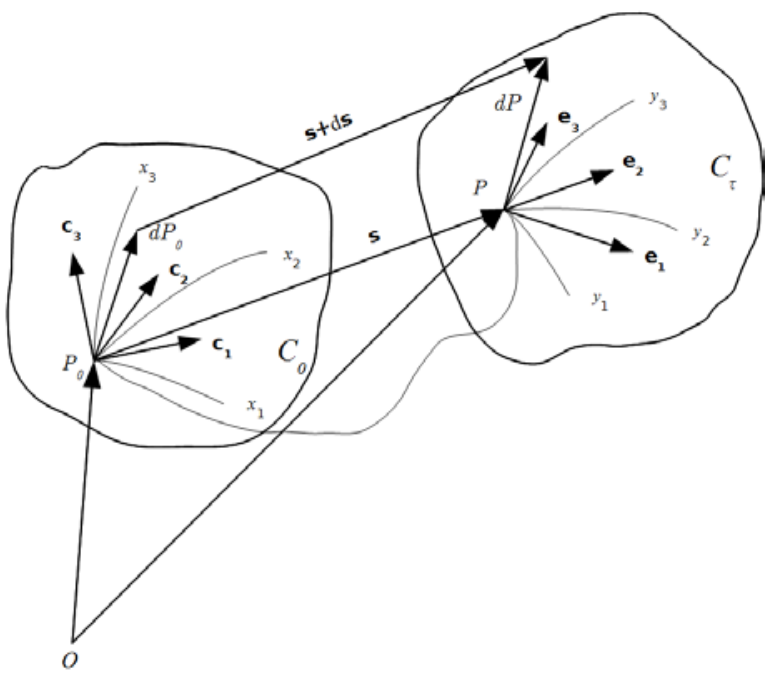

Figure 8. Lagrangian description of time evolution of a continuum.

Therefore, once fixed the initial element of coordinate $x^{\mu}$ the evolution of $y^{\mu}$ is determined uniquely by knowing the proper time (molecular point of view). Without loss of meaning $O$ could coincide with $P_{0}$ whose coordinates for convenience we choose hereafter to be the orthogonal Cartesian ones with respect to the tern $\boldsymbol{c}^{\mu}$ (this is equivalent to refer them to a locally geodetic frame) whence the notation $O P \equiv y^{\mu} \boldsymbol{c}_{\mu}=O P\left(x^{\mu}, \tau\right)$.

Moreover, since we are interested in detectable effects we can also omit the temporal components $x^{0}$ and $y^{0}$ in our reasoning because decoupled from the space coordinates as discussed in the getting the expression for the deformation velocity $K_{\mu \sigma}$ in [1,2], and because the Minkowski tensor is of signature (+,-,-,-) it suffices to change sign of $h_{\mu v}$ according to Eq.(2):

$$
\begin{aligned}
& 2 c \int_{\tau}^{\tau}{ }^{*} K_{\mu \nu}\left(x^{\rho} / \tau^{\prime}\right) d \tau^{\prime} \\
& =\mathrm{g}_{\mu \nu}\left(x^{\rho} / \tau\right)-\eta_{\mu v}=h_{\mu v}\left(x^{\rho} / \tau\right)
\end{aligned}
$$

to work with space distance positive in the next formulas. So, beware here $g_{i j}$ is positive definite .
Therefore, for the line element in $P_{0}$ we have $\mathrm{dl}_{0}^{2}=$ $\mathrm{d} P_{0} \cdot \mathrm{d} P_{0}$, with $\mathrm{d} P_{0}=\left|\mathrm{d} P{ }_{0}\right| \boldsymbol{a}_{0}$, and $\boldsymbol{a}_{0}=a_{0}{ }^{i} \boldsymbol{c}_{\boldsymbol{i}}$ unit vector of $\mathrm{d} P_{0}$. On the other hand, by definition, $\mathrm{d} P{ }_{0}=\mathrm{d} x^{i} \boldsymbol{c}_{\boldsymbol{i}}$ so that

$$
\mathrm{d} x^{i}=\left|\mathrm{d} P_{0}\right| a_{0}^{i}
$$

Similarly, we can write for the line element in $P: \mathrm{dl}^{2}=$ $\mathrm{d} P \cdot \mathrm{d} P$, where $\mathrm{d} P$, referred to the orthogonal tern in $P_{0}$ reads as $\mathrm{d} P=d y^{i} \boldsymbol{c}_{\boldsymbol{i}}=\left(\partial y^{i} / \partial x^{j}\right) \partial x^{j} \boldsymbol{c}_{\boldsymbol{i}}=\boldsymbol{e}_{\boldsymbol{j}} \mathrm{d} x^{j}$ from the relation $\partial O P / \partial x^{j}=\boldsymbol{e}_{\boldsymbol{j}}$. Hence

$$
\left.\mathrm{d} P\right|^{2}=g_{i j} \mathrm{~d} x^{i} \mathrm{~d} x^{j}
$$

We define the physical component of the strain in a given direction as the elongation for unit length in that direction. Taking in account (A.2) e (A.3) we have:

$$
\delta_{a}=\frac{|\mathrm{d} l|-\left|\mathrm{d} l_{0}\right|}{\left|\mathrm{d} l_{0}\right|}=\sqrt{g_{i j} a_{0}^{i} a_{0}^{j}}-1
$$

or through Eq.(2b)

$$
\delta_{a}=\sqrt{1-h_{i j} a_{0}^{i} a_{0}^{j}}-1
$$

i.e. Eq.(17) of [1]. This formula can help us to infer the formula for angle deformation between coordinate axes and to interpret the off-diagonal components of the strain tensor (or tensor of tidal forces if we think of this model); in fact let consider line elements being parallel to axes before deformation, say: $\left.\left(\mathrm{d} l_{1}\right)\right|_{0}=(\mathrm{dx} 1)=\mathrm{dx}^{1}$, $\left.\left(\mathrm{d} l_{2}\right)\right|_{0}=(\mathrm{dx} 2)=\mathrm{dx}^{2}$; after the deformation, by following the reasoning leading to (A.3), we shall have for their scalar product:

$$
\begin{aligned}
& \mathrm{d}_{1} \cdot \mathrm{d} l_{2}=\left|\mathrm{d} l_{1} \| \mathrm{d} l_{2}\right| \cos \Theta_{12}=(\mathrm{d} y 1)^{\mathrm{k}}(\mathrm{d} y 2)_{\mathrm{k}} \\
& =g_{i j}(\mathrm{dx} 1)^{i}(\mathrm{dx})^{j},
\end{aligned}
$$

and because of Eq.(2b) sign changed we get:

$$
\begin{aligned}
& \mathrm{d}_{1} \cdot \mathrm{d}_{2}=\left(-h_{i j}+\delta_{i j}\right)(\mathrm{dx} 1)^{i}(\mathrm{dx})^{j} \\
& =-\left.\left.h_{12}\left(\mathrm{~d} l_{1}\right)\right|_{0} \cdot\left(\mathrm{d} l_{2}\right)\right|_{0} .
\end{aligned}
$$

Finally, on using (A.4) that is to say:

$$
\mathrm{d} l_{1}=\left(\mathrm{d} l_{1}\right) b \sqrt{1-h_{11}}, \quad \mathrm{~d} l_{2}=\left(\mathrm{d} l_{2}\right) b \sqrt{1-h_{22}}
$$

and inserting it into Eq.(A.6) via (A.5) we are able to express

$$
\cos \Theta_{12}=\frac{-\left.\left.h_{12} \cdot\left(\mathrm{d} l_{1}\right)\right|_{0} \cdot\left(\mathrm{d} l_{2}\right)\right|_{0}}{\mathrm{~d} l_{1} \mathrm{~d} l_{2}}=\frac{-h_{12}}{\sqrt{1-h_{11}} \sqrt{1-h_{22}}}
$$

so that if we term $\sigma_{12}=\pi / 2-\Theta_{12}$ the amount to complete a right angle after deformation of coordinate axes $x^{1}$ and $x^{2}$, by taking the sinus function of $\sigma$ we obtain the (A.8) again, and therefore:

$$
\sin \sigma_{12}=\cos \Theta_{12} \Leftrightarrow \sigma_{12} \approx-h_{12}
$$

namely the decreasing of angle between axes direction is related to so called the shearing strain or off-diagonal element of strain tensor. To conclude we show in Figure 9 the effect of the presence of shearing strains $h_{21}=h_{12}$ $=A_{\otimes} \cos (\tau)$ on an unit vector according to Eq.(A.4) disregarding angular axes deformation: it is plain how cylindrical symmetry gets broken. Of course, $\oplus$ polarized 
$h_{\mu v}$ produces in-phase elongations in the direction of

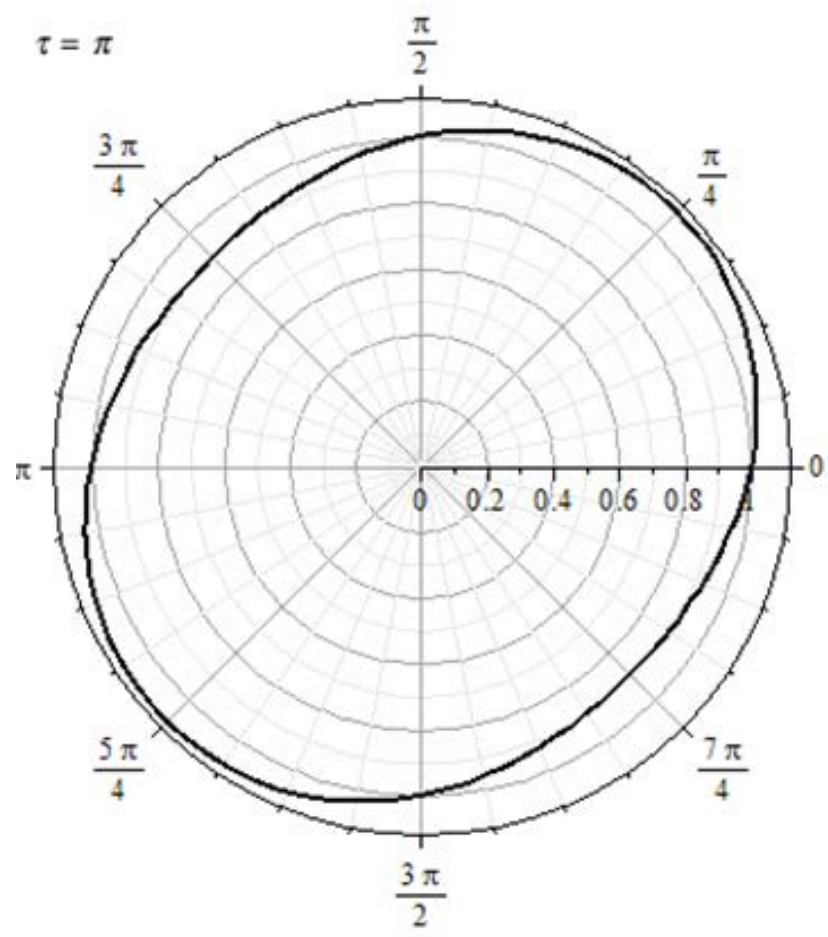

coordinate axes as shown in [1] according (A.7).

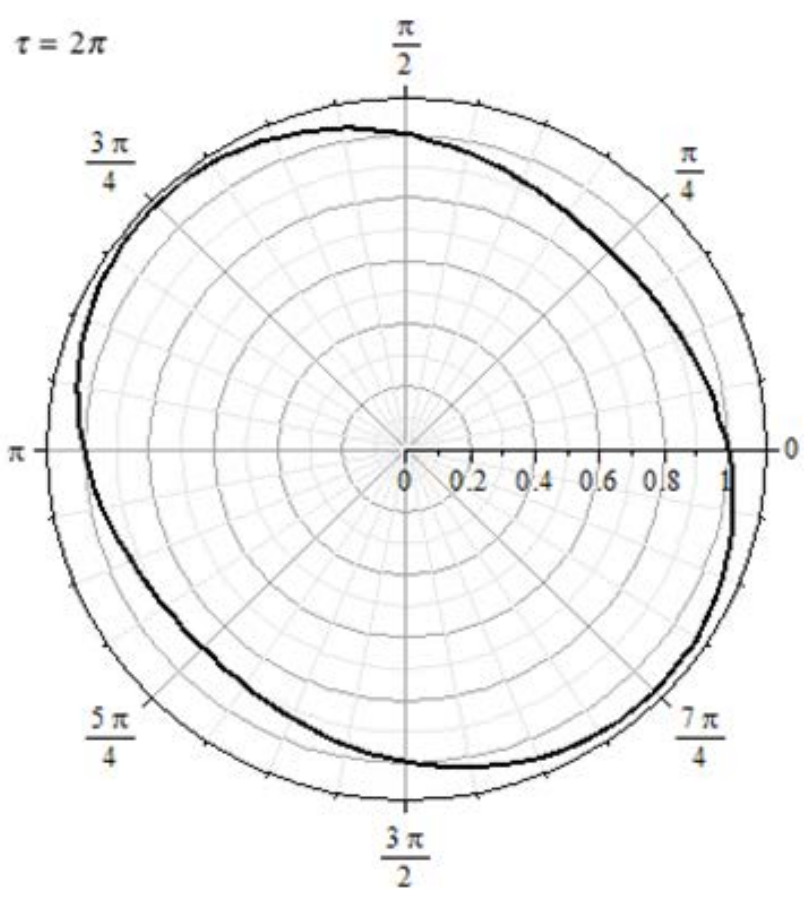

Figure 9. Polar diagram of the elongation of a vector on the unit circle subject to $\otimes$ deformation according Eq.(A.4) for $|\mathrm{d} l|, a_{0}=\left(\begin{array}{c}\cos \theta \\ \sin \theta\end{array}\right)$. Angular shrinking of axes has been left out. The effect of deformation is manifestly exaggerated for understanding, actually being for instance of the order $1 \mathrm{E}-21$ for B1913+16

\section{Appendix B: Action of the model and a cartesian derivation of main equations}

We can infer the main equations of the Vortex Model from a variational action principle in a form remembering that for the coupling of charged matter current interacting with an electromagnetic field:

$$
\begin{aligned}
& A=\int L d^{4} x^{\prime} \\
& =\int d^{4} x^{\prime}\left[\begin{array}{c}
-\frac{1}{4} \Phi_{v \sigma \mu} \Phi^{v \sigma \mu}-K_{\mu \sigma}\left(\begin{array}{l}
K^{\mu}{ }_{\alpha} R^{\alpha \sigma} \\
-K_{\beta}^{\alpha} R_{\alpha \tau}^{\sigma \mu} g^{\beta \tau} \\
{C^{\mu}{ }_{v} / \sigma \gamma}^{4 S}
\end{array}\right) \\
+\frac{K_{\gamma \delta} g^{\alpha \gamma} R^{\beta \delta} K_{\alpha \beta}}{2}-\frac{K_{\gamma \delta} R^{\alpha \delta \beta \gamma} K_{\alpha \beta}}{2}
\end{array}\right]
\end{aligned}
$$

whereupon the strength tensor $\Phi_{\mu v \sigma}=\nabla_{\mu} K_{v \sigma}-\nabla_{v} K_{\mu \sigma}$ in which $K_{\mu v}$ play the role of the field potential as in electromagnetism and the second term in brackets the matter source term. From that the EM tensor in free space reads:

$$
t_{\mu}{ }^{v}=-\Phi_{\mu}{ }^{\sigma \alpha} \Phi_{\sigma \alpha}^{v}+1 / 4 \delta_{\mu}{ }^{v} \Phi_{\alpha \sigma \rho} \Phi^{\sigma \alpha \rho}
$$

We already know the variation leads to wave equation in presence of matter as seen in the reference paper [6]. By the way, let us show that the classical deformation speed $1 / 2 \partial_{\tau} g_{\mu v}$ corresponds to the deformation tensor of mechanics. Defined the local basis of vectors at each point of the cronotope $\boldsymbol{e}_{\alpha}=\partial O P / \partial x^{\alpha}$, differentiating $\boldsymbol{e}_{\mu}\left(x^{v} / \tau\right)$ with respect to $x^{v}$, we get for definition of Christoffel symbols : $\partial_{v} \boldsymbol{e}_{\mu}=\Gamma_{v \mu}^{\alpha} \boldsymbol{e}_{\alpha}$. From that, it turns out that the gradient of the 4-velocity $\partial_{v} v=\partial_{v}\left(v_{\mu} e^{\mu}\right)=\left(\partial_{v} v_{\mu}\right.$ $\left.\Gamma_{v \mu}^{\alpha} v_{\alpha}\right) \boldsymbol{e}^{\mu}=\left(\nabla_{v} v_{\mu}\right) \boldsymbol{e}^{\mu}$ leading $K_{\mu v}=\partial_{\tau} g_{\mu v}=\partial_{\tau}\left(\boldsymbol{e}_{\mu} \cdot \boldsymbol{e}_{v}\right)=$ $1 / 2\left(\partial_{\mu} \boldsymbol{v} \cdot \boldsymbol{e}_{v}+\partial_{v} \boldsymbol{v} \cdot \boldsymbol{e}_{\mu}\right)$ to the classical expression: $K_{\mu v}=1 / 2\left(\nabla_{\mu} v_{v}+\nabla_{v} v_{\mu}\right)$.

We know that although the vortex and the deformation tensor are both independent, they are differentially bound through the Vorticity formula of continua that we now derive in 3 dimension cartesian coordinates as due to Zhen $\mathrm{Li}$ (Tsinghua Univ.), addressing the reader to the more thorough approach of Ferrarese and Stazi [4]. Standing the definition of vorticity:

$$
\omega_{h k}=1 / 2\left(\epsilon_{h k s} \omega^{s}\right)(h k s=1,2,3)
$$

the Jacobi relation turns out:

$$
\begin{aligned}
& \nabla_{l} \omega_{h k}+\nabla_{k} \omega_{l h}+\nabla_{h} \omega_{k l} \\
& =1 / 2\left(\epsilon_{h k s} \omega_{/ l}^{s}+\epsilon_{l h s} \omega_{/ k}^{s}+\epsilon_{k l s} \omega_{/ h}^{s}\right) \\
& =1 / 2 \omega_{/ s}^{s}=1 / 2 \operatorname{div} \omega_{\square}=1 / 4 \operatorname{div}(\operatorname{curl}(v))=0 .
\end{aligned}
$$

Given that, then we have:

$$
2 \nabla_{l} \omega_{h k}=\nabla_{l}\left(\nabla_{h} v_{k}-\nabla_{k} v_{h}\right)=\nabla_{h} \nabla_{l} v_{k}-\nabla_{k} \nabla_{l} v_{h}
$$

and remembering that $\nabla_{h} v_{k}=q_{h k}=K_{h k}+\omega_{h k}$ :

$$
\begin{aligned}
& =\nabla_{h} q_{l k}-\nabla_{k} q_{l h}=\nabla_{h} K_{l k}-\nabla_{k} K_{l h}+\nabla_{h} \omega_{l k}-\nabla_{k} \omega_{l h} \\
& =\nabla_{h} K_{l k}-\nabla_{k} K_{l h}+\nabla_{l} \omega_{h k}
\end{aligned}
$$

hence the vorticity formula for its gradient: 


$$
\nabla_{l} \omega_{h k}=\nabla_{h} K_{l k}-\nabla_{k} K_{l h}
$$

which holds in V4 general coordinates as well. We make notice that in the definition of vorticity and deformation tensors $([1,6])$, both sides of previous equation are scaled by the speed of light $c$ in order to introduce the constant $S$ of dimensions of [lenght] ${ }^{-1}$, whence the ansatz: $C_{\mu v}=S \omega_{\mu v}$, $C_{\mu v}=R_{\mu \nu \rho \sigma} \epsilon^{\rho \sigma}$, so we have the main equation:

$$
\nabla_{\sigma} C_{\mu v}=S\left(\nabla_{\mu} K_{v \sigma}-\nabla_{v} K_{\mu \sigma}\right)(\mu, v, \sigma=1,2,3,4) \text {. }
$$

\section{Acknowledgement}

I'm grateful to CINECA supercomputing section for supplying its 192Gb RAM HP-DL980 hardware.

\section{References}

[1] Antonelli,S., "Outstanding Outcomes from a Recent Theory of Gravity”, International Journal of Physics, Sciepub, 2,6,2014, pp.267-276, 2014. [Online] Available:

http://www.sciepub.com/portal/downloads?doi=10.12691/ijp-2-610\&filename=ijp-2-6-10.pdf.

[2] Landau, L., Lifchitz, E., The Classical Theory of Fields, vol.2, sec.82, Butterworth-Heinemann, 1975.

[3] Loinger,A., "Gravitational waves are fictitious entities II", http://xxx.lanl.gov/abs/astro-ph/9904207, 1999.

[4] Ferrarese,G., Stazi, L., Lezioni di Meccanica Razionale , vol 2 chap.VIII, §1.10, Pitagora Eds, Bologna, 1989 .

[5] Ferrarese, G., Bini, G.., Introduction to relativistic continuum mechanics, Springer, Heidelberg, 2008.
[6] Tailherer, M., "A Critical Reading on the Theory of Gravitational Wave Propagation”, Scientific Journals, Journal of Physical \& Natural Sciences 1, 1, 2007.

[7] Gershtein, S.S., Logunov, A.A.,Mestvirishvili, M.A., “The upper limit on the graviton mass", 1997. [Online]. Available: arXiv:hepth/9711147.

[8] Visser, M., "Mass for the Graviton”,1998, arXiv:gr-qc/9705051.

[9] Ohanian, H.C., Ruffini, R., Gravitation and Spacetime, Chap.5 §5.2, W.W. Norton \& Company, 1994.

[10] Sokolnikoff, I.S., Tensor Analysis, Chap. 6, §105, Wiley, New York, 1951.

[11] Finn, L.S., Sutton, P.J., "Bounding the mass of the graviton using binary pulsar observations”, Phys. Rev. D65, 044022, 2002. [Online]. Available: arXiv:gr-qc/0109049.

[12] Will, C.,W., "Bounding the mass of the graviton using gravitational-wave observations of inspiralling compact binaries. Phys. Rev. D57, 2061. (1998) [Online]. Available: arXiv:grqc/9709011.

[13] Maggiore, M., Gravitational waves, vol. 1, §6.2.3, OUP Oxford, 2007.

[14] Weisberg, J.M.,Taylor, J.H., "Relativistic Binary Pulsar B1913+16: Thirty Years of Observations and Analysis",2004 . [Online] . Available: arXiv:astro-ph/0407149 v1

[15] Antonelli. S., "Importance of the Gauss' 2nd differential form in Relativity”, viXra e-Print archive, 2013, [Online]. Available: http://www.vixra.org/abs/1310.0190.

[16] Kramer, M., et al.,“Tests of general relativity from timing the double pulsar”, 2006, [Online]. Available: arXiv:0609417 [astro$\mathrm{ph}]$.

[17] Wolszczan, A., "A nearby 37.9-ms radio pulsar in a relativistic binary system”, Nature, 350, 688 ,1991.

[18] Blanchet,L., "Post-Newtonian theory and the two-body problem", 2010. [Online]. Available: arXiv.org/gr-qc/0907.3596

[19] Jacoby, B. A, et al. "Measurement of orbital decay in the double neutron star binary B2127+11C", The Astroph. J., 644, L113L116, 2006. [Online]. Available: arXiv:astro-ph/0605375. 